\title{
Estudo de viabilidade de rede de bibliotecas em nuvem da administração pública federal
}

\author{
Elaine Coutinho Marcial \\ elamarcial@gmail.com \\ Instituto de Pesquisa Econômico Aplicada, Brasília, DF, Brasil \\ Cristine Coutinho Marcial Pinheiro \\ cristine.marcial@gmail.com \\ Ministério do Planejamento Orçamento e Gestão, Brasília, DF, Brasil \\ Maria Elenita M. Nascimento \\ elenitan@gmail.com \\ Universidade de Brasília, Centro de Excelência em Turismo, Brasília, DF, Brasil \\ João Tribouillet Marcial de Menezes \\ jotatri@gmail.com \\ Universidade de Brasília, Faculdade de Tecnologia, Brasília, DF, Brasil
}

Resumo: O Cloud Computing é uma alternativa para as bibliotecas da Administração Pública Federal Direta em função do crescimento da produção de documentos digitais. Entretanto, é necessária infraestrutura que dê suporte a esse modelo. Nesse contexto, o objetivo geral do estudo é identificar se a Administração Pública Federal Direta está preparada para lidar com a explosão informacional digital por meio do Cloud Computing, assegurando que os registros bibliográficos das publicações oficiais sejam preservados, em formato digital, e acessível para as gerações futuras. Pesquisa descritiva cujo método utilizado foi a análise de cluster. Os dados foram coletados por meio de levantamento estatístico do comportamento das variáveis do modelo proposto. O universo pesquisado contemplou os 24 ministérios e a Casa Civil, sediados em Brasília. Como resultado, foram identificados quatro clusters distintos e ficou evidenciado que a maioria dessas bibliotecas não está preparada para a implantação de bibliotecas em cloud computing, necessitando de diversos investimentos tanto em infraestrutura informacional digital e tecnológica, quanto em pessoal capacitado com perfil multidisciplinar, bem como recursos para implantação e custeio.

Palavras-chave: Biblioteca digital, Cloud Computing, Ciência da Informação.

\section{Network feasibility study libraries in the cloud for the public administration federal}

Abstract: The Cloud Computing is an option for the direct federal public administration libraries due to the increased production of digital documents. However, an infrastructure is necessary to support this model. In this context, this research aim is to identify if the direct federal public administration is prepared to deal with the digital information explosion by using cloud computing, to guaranty that the bibliographic record of the official publications could be preserved and accessible, in digital format, for future generations. It was a descriptive research that used cluster analysis. The data were collected by a statistical survey to understand the variable behavior in the proposed model. The population were the 24 ministries and the Civil House, all of them in the city of Brasilia-DF. We identified four distinct clusters and it was evident that most of these libraries were not prepared to implement cloud computing libraries, requiring budget for several investments: in digital and technological information 
infrastructure, in training persons with multidisciplinary skills and knowledge, for implementation and to defray.

Keywords: Digital library, Cloud Computing, information science.

\section{Estudio de viabilidad de una red de bibliotecas en las nubes para la administración pública federal}

Resumen: El Cloud Computing es una alternativa para las bibliotecas de la Administración Pública Federal Directa en función del crecimiento de la producción de documentos digitales. Sin embargo, es necesaria la infraestructura que dé soporte a ese modelo. En ese contexto, el objetivo general del estudio es identificar si la Administración Pública Federal Directa está preparada para lidar con la explosión de información digital por medio del Cloud Computing, asegurando que los registros bibliográficos de las publicaciones oficiales sean preservados, en formato digital, y accesibles para las generaciones futuras. La investigación es descriptiva y el método utilizado fue el análisis de clusters. Los datos fueron colectados por medio del levantamiento estadístico del comportamiento de las variables del modelo propuesto. El universo investigado contempló los 24 ministerios y la Casa Civil, con sede en Brasilia. Como resultado, fueron identificados cuatro clusters que se distinguían y quedó en evidencia que la mayoría de esas bibliotecas no están preparadas para la implantación de bibliotecas en cloud computing, necesitando de diversas inversiones tanto en infraestructura en información digital y tecnológica, como de personal capacitado con perfil multidisciplinar, así como de recursos para su implementación y costeo.

Palabras clave: Biblioteca Digital, Cloud Computing, Ciencias de la Información. 


\section{Introdução}

Cloud Computing (computação em nuvem) é definido como um modelo no qual a computação (processamento, armazenamento e software) encontra-se em algum lugar da rede e pode ser acessada remotamente, via internet, com o mínimo de gerenciamento. A perspectiva é que no futuro não será necessário instalar nenhum software nos computadores para executar qualquer tipo de tarefa, como edição de imagens e vídeos até a utilização de programas de escritório (Office), uma vez que todos poderão ser acessados por meio da internet.

São muitas as vantagens deste modelo dentre elas podemos citar: a) muitos aplicativos, arquivos e outros dados relacionados não necessitarão ser instalados ou armazenados no computador do usuário ou em servidor próximo, uma vez que esse conteúdo passa a ficar disponível nas nuvens, ou seja, na internet; b) compartilhamento de dados e trabalho colaborativos se tornará mais fáceis, uma vez que todos os usuários acessarão as aplicações e os dados do mesmo lugar - a nuvem; c) o usuário poderá contar com melhor controle de gastos uma vez que muitas das aplicações são gratuitas e quando necessário pagar o usuário só o fará em relação aos recursos que usar ou pelo tempo de utilização.

Diferentemente do passado, hoje, as bibliotecas lidam cada vez mais com informação virtual. Antes, se limitavam a explorar o potencial dos computadores pessoais em seus ambientes, hoje interagem com soluções baseadas em tecnologias criando um grande desafio no gerenciamento desses recursos informacionais. Nesse contexto, a Cloud Computing proporciona às bibliotecas virtuais serviços mais dinâmicos, flexíveis e escalonáveis.

Destaca-se que esse recurso tecnológico já influencia eventos na área da biblioteconomia, como a Conferência Anual da American Library Association (ALA), que vem promovendo, nos últimos anos, sessões voltadas à computação em nuvem para serviços de biblioteca, tais como: o modelo de gestão dos serviços e aplicativos hospedados; as propostas inovadoras na abordagem dos serviços tradicionais de informação migrados para ambientes digitais; e as perspectivas vislumbradas pela biblioteca e os riscos a serem evitados na nova plataforma (PASQUI, 2010).

A existência de uma biblioteca digital facilita a utilização da plataforma de computação em nuvem, pois seu acervo já está digitalizado. Além disso, a biblioteca em nuvem oferece soluções para a falta de conhecimento técnico ou para uma equipe de sistemas de pequeno porte. 
Buscou-se nesta pesquisa responder a seguinte questão: a administração pública federal direta, representada por seus ministérios, está preparada para lidar com a explosão do volume informacional digital por meio do Cloud Computing?

Nesse contexto, o objetivo geral do estudo é identificar se a administração pública federal direta está preparada para lidar com a explosão informacional digital por meio do Cloud Computing, assegurando que os registros bibliográficos das publicações oficiais sejam preservados e acessíveis, em formato digital, para as gerações futuras. A presente pesquisa se desdobra nos seguintes objetivos específicos:

- Verificar se existe uma infraestrutura informacional propicia para o funcionamento das bibliotecas "em nuvem".

- Verificar se há infraestrutura de TI, incluindo plataforma, infraestrutura e software como serviços para o funcionamento das bibliotecas "em nuvem".

- Identificar se há pessoal capacitado para trabalhar com as bibliotecas "em nuvem".

- Verificar se há recursos financeiros para implantação e custeio das bibliotecas "em nuvem".

Visando alcançar esses objetivos foi realizada uma pesquisa/levantamento estatístico do comportamento das variáveis do modelo proposto, para institucionalização de bibliotecas em nuvem. A pesquisa foi do tipo censo, tendo utilizado como instrumento de pesquisa o questionário. Foram aplicados métodos estatísticos descritivos aos dados qualitativos obtidos. O universo pesquisado contemplou os 24 ministérios e a Casa Civil, sediados em Brasília.

\section{Revisão da Literatura}

A Ciência da Informação é interdisciplinar, tendo como áreas fundamentais a Biblioteconomia e a Ciência da Computação, conforme argumentado por Saracevic (1995). Nesse contexto, a Ciência da Informação engloba pesquisas, tanto no campo científico, quanto no aplicado, que muitas vezes, conjugam a inter-relação entre a Ciência da Computação e os processos informacionais, principalmente os que se referem ao armazenamento, organização, produção e recuperação da informação.

Atualmente, com o advento das Tecnologias da Informação e da Comunicação (TIC), essa relação se faz muito mais forte, e os estudos tornam-se mais necessários. Isso porque, há um permanente crescimento da produção informacional em meio digital, seja ela científica ou não, e da oferta de aplicativos e dispositivos digitais, cada vez mais amigáveis, que surgem para facilitar os diversos processos informacionais, independente do formato da informação. 
É nesse contexto que surge a tecnologia de CLOUD Computing, traduzida equivocadamente para o português como "computação em nuvem". Isso porque CLOUD é um acrônimo de: (1) C - Computing resources; (2) L - that is Location independent, (3) $\mathbf{O}$ - can be accessed via Online means; (3) U - used as an Utility; (4) D - is available on Demand (YUVARAJ, 2013). Ou seja, CLOUD representa um recurso informacional que funciona como um utilitário por meio de acesso on-line armazenado em um local independente e disponível por demanda. São exemplos o Facebook, Twitter, Flickr, YouTube, os provedores de email, como Google, os armazenadores de informação como o Google Docs e o Dropbox.

Recentemente, os pesquisadores Mayank Yuvaraj e Ajay Pratap Singh (2012) realizaram uma vasta revisão da literatura sobre a relação entre Cloud Computing e bibliotecas, por meio da consulta a diversas bases de dados. Segundo esses autores, o conceito e uso de CLOUD Computing datam de 1960, entretanto, somente despontam como realidade, a partir de 2007. Há diversas definições para CLOUD Computing como as de Boss, Malladi et al. (2007), Heyes (2008), Plummer et al. (2008), Voas e Zhang (2009), Murley (2009) e Madhubala (2012).

O CLOUD Computing é visto como um novo paradigma focado no fornecimento de serviços de compartilhamento de dados e sistemas computacionais, por meio da internet, que se utiliza de estruturas complexas (MADHUBALA, 2012; PLUMMER et al., 2008).

Ele permite a virtualização, em grande escala, de infraestrutura representada por um pool compartilhado e recursos computacionais configurados, como redes, servidores, armazenamentos e aplicativos capazes de fornecer o suporte necessário para acesso a serviços informacionais convenientes e sob demanda a distância para seus usuários. Isso tudo com o mínimo de gerenciamento (BOSS; MALLADI et al., 2007; MELL; GRANCE, 2009).

Segundo Mell e Grance (2009), o modelo CLOUD, possui cinco características essenciais, três modelos de serviço e cinco modelos de implantação:

- Características essenciais: (1) por demanda e self-service; (2) acesso amplo a rede; (3) pool de recursos, como: armazenamento, processamento, memória, rede banda largura e máquinas virtuais para atender a múltiplos consumidores; (4) rápida elasticidade de provisionamento; e (5) serviço de controle e aprimoramento do uso dos recursos automaticamente.

- Modelos de serviços: (1) SaaS (software as a service); PaaS (Plataform as a Service); (3) laaS (Infrastructure as a Service). Esses modelos também foram destacados por: Murley (2009); Yuvaraj e Singh (2012); Silva e Mucheroni (2013); e Yuvaraj (2013). 
- Modelos de implantação: os modelos de CLOUD podem ser: (1) privado; (2) comunitário; (3) público; e (4) híbrido, conjugando os já citados. Esses modelos também foram citados por: Yuvaraj e Singh (2012); e Silva e Mucheroni (2013).

Outro movimento observado em todo o mundo é o crescimento da digitalização e da produção digital de documentos. Nesse contexto, as bibliotecas tradicionais foram impulsionadas a desenvolverem bibliotecas digitais, para o gerenciamento do crescente acervo digital. O próximo passo dessa evolução tecnológica foi a adoção, pelas bibliotecas digitais, do CLOUD Computing, criando o que vem sendo chamado de "bibliotecas em nuvem". Esse movimento foi impulsionado pelas vantagens que o CLOUD Computing oferece, como a redução de custos e facilidade de implantação e uso das tecnologias disponíveis para gestão da informação digital. Destaca-se que os serviços baseados em "nuvem" transformam a forma como as bibliotecas funcionam, disponibilizando mais tempo para que os profissionais da informação possam oferecer melhores serviços aos usuários (SHOWER, 2011).

Entretanto, para a implantação de uma "biblioteca em nuvem" há a necessidade da existência de um acervo digital (infraestrutura informacional) e de competências informacional e tecnológica (infraestrutura de pessoal e tecnológica) por parte de seus gestores (YUVARAJ; SINGH, 2012). Isso porque a implantação e manutenção de uma "biblioteca em nuvem" pressupõe a adoção de serviços oferecidos pelas TIC por parte da biblioteca ou centros de documentação e informação.

A utilização da "biblioteca em nuvem" traz diversos benefícios, como: escalabilidade, fácil implantação, uso e gestão sem a necessidade de grandes conhecimentos tecnológicos, maior grau de flexibilidade dos serviços oferecidos, uso de recursos especializados; melhoria da qualidade dos serviços e redução dos custos de manutenção (YUVARAJ; SINGH, 2012). Entretanto, alguns autores (SHOWER, 2011; YUVARAJ; SINGH, 2012) destacam como vulnerabilidades ou pontos fracos da adoção desse modelo às questões ligadas à segurança, à privacidade, à confiança e à perda do controle de danos internos, cujos riscos precisam ser gerenciados caso seja adotado tal modelo. Sendo assim, a partir do gerenciamento de seus riscos, o CLOUD Computing mostra-se como opção de infraestrutura tecnológica para o momento atual vivido pelas bibliotecas que necessitam lidar com acervos digitais.

No que diz respeito à infraestrutura informacional, a implantação de uma plataforma de CLOUD Computing em uma biblioteca é facilitada se esta já dispuser de uma biblioteca digital, pois o acervo digital já existe. Uma biblioteca digital pode ser definida, de maneira bastante ampla, como uma coleção organizada de objetos digitais, associada a um conjunto de serviços acessíveis em ambientes distribuídos visando atender às necessidades de 
comunidades de usuários. É um dos tipos mais avançados e complexos de sistemas de informação, por envolver, dentre outras coisas, busca estruturada, navegação, recomendação, preservação de documento, serviços de informação multimídia e disseminação seletiva da informação (TOFFOLI, 2012).

A Ciência da Informação prevê a descrição de objetos digitais, como uma maneira de possibilitar o armazenamento e a recuperação da informação (ARELLANO, 2006). Partindo da definição geral que objeto digital é um objeto de informação, de qualquer tipo e formato expressa sob a forma digital. Thibodeau (2002) descreve os objetos digitais como sendo um objeto físico, lógico e conceitual. Sendo assim, apresenta propriedades diferentes em cada um desses níveis. Por exemplo: a) objeto físico - é uma inscrição de sinais em um meio físico; b) objeto lógico - é reconhecido e processado por software; c) objeto conceitual - é reconhecido e entendido por uma pessoa ou reconhecido e processado por um software capaz de executálo.

Destaca-se que a "publicação em meio digital, de livre acesso, sem dúvida é mais democrática, mais fácil de intercambiar e disseminar o conhecimento", conforme analisado pela coordenadora da Biblioteca Nacional Digital, Ângela Bettencourt (AVANCINI, 2014).

O principal desafio do suporte digital é assegurar a preservação das publicações digitais indefinidamente ao longo do tempo, de modo que as futuras gerações tenham acesso à memória institucional. Na opinião de Humberto Innarelli, pesquisador e analista de desenvolvimento de sistemas do Arquivo Edgard Leuenroth da Universidade Estadual de Campinas (Unicamp), esse é um dos principais riscos que se corre com a disseminação das TIC (AVANCINI, 2014).

Entretanto, infraestrutura informacional também contempla a existência de um controle bibliográfico da produção institucional, associado à disponibilização desse conteúdo em meio digital. Esse controle bibliográfico tem como objetivo controlar o registro da publicação oficial para preservação da memória de uma organização. Consiste de técnicas e procedimentos de identificação, localização, obtenção e registro sistemático da produção intelectual de uma organização para torná-la acessível a quem necessitar dela (SANTOS, 2013). No contexto das publicações oficiais, a institucionalização do controle bibliográfico garante o controle do registro da publicação oficial para preservação da memória das organizações públicas (BRASIL, 2010).

As principais vantagens do controle bibliográfico da produção institucional é assegurar a transparência, garantir a preservação da memória institucional e sua visibilidade institucional, disponibilizar melhores serviços otimizando recursos (SANTOS, 2013). Cabe 
destacar que na era digital, esse controle bibliográfico abrange todo tipo de elemento capaz de armazenar informação como: livros, periódicos, apresentações, vídeos etc. (BRASIL, 2010).

Quanto à infraestrutura de pessoal, destaca-se a necessidade de uma equipe multidisciplinar de profissionais da informação, como cientistas de informação, bibliotecários, arquivistas e profissionais da tecnologia da informação, que sejam capazes de: trabalhar em equipe; gerir os diversos tipos e formatos de informação digital; desenvolver e produzir serviços informacionais a serem difundidos por meio dos dispositivos digitais; bem com deter conhecimento dos fluxos informacionais e ser capazes de lidar com os usuários digitais (SOUTO, 2006; BELLUZZO, 2011).

Para Souto (2006), existe um novo perfil de competências para o profissional da informação no contexto social atual. Ele deve ser um sujeito com permanente capacidade de aprendizagem e de adaptação às mudanças, deve saber trabalhar em grupo, de referência em equipes multidisciplinares, e ter domínio da linguagem das máquinas, ou seja, ser alfabetizado digitalmente. Além disso, deve saber estar em constante processo de busca e desenvolvimento - aprender a aprender - para poder adaptar-se aos novos modelos organizacionais e de gestão do trabalho e ser um agente do processo de inovação.

Focando esse contexto no ambiente da administração pública federal, verifica-se que o governo brasileiro possui uma série de publicações oficiais, que são definidos como:

Todo e qualquer documento impresso, sonoro ou visual, armazenado eletronicamente ou por qualquer outro meio, produzido e editado por qualquer processo gráfico ou eletrônico, convencional ou não, sob a responsabilidade, às expensas, por ordem ou com a participação dos órgãos da administração pública, direta, indireta, autarquia ou fundacional, dos poderes Executivo, Legislativo e Judiciário, nos níveis federal, estadual, municipal e distrital, refletindo a opinião oficial ou a vontade e as atividades do governo (BRASIL, 2010).

A Administração Pública Federal tem a preocupação e o zelo com o recolhimento, a organização, a guarda, a preservação e a divulgação das publicações editadas pelas Instituições Federais. Toda publicação produzida em território Nacional, por qualquer meio ou suporte, tem como objetivo assegurar a coleta, a guarda e a difusão da produção intelectual brasileira, visando à preservação e a formação da Coleção Memória Nacional (BRASIL, 2010).

Além da importância intrínseca da preservação das publicações oficiais, a partir de 2011, com a criação da Lei de Acesso a Informação (Lei no 12.527 de 18/11/2011), torna-se obrigatório às instituições públicas garantirem o direito constitucional de acesso dos cidadãos às informações públicas. A nova Lei evidencia a necessidade da existência de uma forma adequada de armazenar e recuperar as publicações digitais, para que elas não se "percam" nos 
diversos sítios oficiais ou venham a desaparecer por ocasião de reformulações futuras desses referidos sítios (SANTOS, 2013; BRASIL, Lei no 12.527, 2011).

Sendo assim, com base na revisão da literatura, conclui-se que para a implantação de "bibliotecas em nuvem" das publicações oficiais editadas ou produzidas pela Administração Publica Federal Direta, é necessário o desenvolvimento de: (1) uma infraestrutura informacional - publicações oficiais em meio digital e um controle bibliográfico dessas publicações como forma de depósito legal. (2) uma infraestrutura tecnológica; e (3) uma infraestrutura de pessoal (capacitada nas áreas de informação, como: cientistas da informação, bibliotecários, arquivista e profissionais de tecnologia da informação, com capacidade de trabalho em equipe). Destaca-se também a necessidade de recursos financeiros para investimento e custeio na "biblioteca em nuvem", pois, mesmo sendo mais baratos, os serviços de SaaS, PaaS e laaS, disponibilizados pelo COULD Computing às bibliotecas e centros de documentação, são pagos.

\section{Metodologia}

A pesquisa classifica-se como exploratória, cujo método foi o levantamento estatístico do comportamento das variáveis do modelo proposto para institucionalização de bibliotecas em nuvem. A pesquisa foi do tipo censo cujo instrumento de pesquisa foi o questionário. Foram aplicados métodos estatísticos descritivos aos dados qualitativos obtidos. 0 Quadro 1 apresenta a relação entre os objetivos da pesquisa e as variáveis do modelo.

Quadro 1 - Objetivos e Variáveis da pesquisa

\begin{tabular}{|l|c|}
\hline \multicolumn{1}{|c|}{ Objetivos } & Variáveis \\
\hline $\begin{array}{l}\text { Identificar se a administração pública federal direta está } \\
\text { preparada para lidar com a explosão informacional digital } \\
\text { por meio da computação em nuvem. }\end{array}$ & v11_volinf \\
\hline $\begin{array}{l}\text { Verificar se há uma infraestrutura informacional propicia } \\
\text { para o funcionamento da biblioteca "em nuvem". }\end{array}$ & $\begin{array}{c}\text { v4_bidig, v5_cobibli, } \\
\text { v6_publiof }\end{array}$ \\
\hline $\begin{array}{l}\text { Verificar se há infraestrutura de TI, incluindo plataforma e } \\
\text { software como serviço, para o funcionamento da biblioteca } \\
\text { "em nuvem". }\end{array}$ & $\begin{array}{c}\text { v8_senuv, v9_tipos, } \\
\text { v12_tic, }\end{array}$ \\
\hline $\begin{array}{l}\text { Identificar se há pessoal capacitado para trabalhar nas } \\
\text { bibliotecas em nuvem. }\end{array}$ & v7_rh \\
\hline $\begin{array}{l}\text { Verificar se há recursos financeiros para implantação e } \\
\text { manutenção da biblioteca em nuvem. }\end{array}$ & v10_inv \\
\hline
\end{tabular}

Fonte: Produção dos autores (2014). 
As variáveis que compõe o modelo, listadas na no Quadro 1, possuem os seguintes significados, levantados por meio de aplicação de questionário:

v4_bidig - Existência ou não de biblioteca digital, no caso de não existir: se há ou não previsão de investimento ou se existem estudos preliminares.

v11_volinf - Identifica se o ministério está preparado para lidar com a explosão do volume de informação digital por meio da computação "em nuvem". Caso não esteja, se está se preparando ou ainda não.

v3_bibli - Verifica se há biblioteca ou centro de documentação no órgão. Variável utilizada para definir a população da pesquisa.

v5_cobibli - Se existe ou não controle bibliográfico. No caso de não existir, se há ou não previsão de investimento, ou se existem estudos preliminares.

v6_publiof - Identifica se as publicações oficiais do ministério estão armazenadas em bibliotecas digitais, no caso de não existir: se há ou não previsão de investimento ou se existem estudos preliminares.

v8_senuv - Existência ou não de serviços informacionais em nuvem, no caso de não existir: se há ou não previsão de investimento.

v9_tipos - Caso existam serviços informacionais em nuvem, identifica quais são esses serviços. v12_tic - Identifica se o biblioteca do ministério compartilha, em nuvem, a infraestrutura de tecnologia da informação e comunicação.

v7_rh - Identifica se há recursos humanos preparados para trabalhar com bibliotecas "em nuvem".

v10_inv - Identifica se há previsão de investimento permanente para ampliação de espaço de armazenamento em função da crescente quantidade de documentos digitais para a preservação da memória institucional.

A pesquisa teve início em junho de 2014, quando foi realizado pré-teste do questionário, e a aplicação do questionário foi concluída em final de agosto de 2014. O universo pesquisado contemplou os 24 ministérios e a Casa Civil, sediados em Brasília - DF (cuja lista encontra-se em: <http://www.pgr.mpf.mp.br/outros-sites/poder-executivo>), que fazem parte da estrutura da Administração Pública Federal Direta, do Poder Executivo Federal.

Não participaram da pesquisa as Secretarias ligadas a Presidência da República nem órgãos de Assessoramento da Presidência da República que possuem status de exceto a Biblioteca da Casa Civil.

Foi realizado levantamento preliminar e descobriu-se que, dos 24 ministérios foco da pesquisa, seis não possuem biblioteca ou centro de documentação. Sendo assim, a população 
da pesquisa foi reduzida para 18 ministérios e a Biblioteca da Casa Civil. Foram encaminhados questionários para todas as bibliotecas e centros de documentação dessas organizações considerado restrição da pesquisa -, entretanto, somente 16 responderam que representa uma amostra de $84,2 \%$ da população.

A seguir, serão apresentados os principais resultados do levantamento. Para tanto, foi utilizado o software estatístico SPSS para a realização das análises estatísticas. Os dados foram agrupados em tabelas de frequência, que apresentam os resultados nos formatos absolutos e relativos. Foi realizado cruzamento entre variáveis, calculado a medida de posição "moda" e por fim foi realizada a classificação da amostra por meio da utilização de análise de cluster pelo método hierárquico. Por fim foi realizado analise de cluster, por meio da utilização do modelo de classificação hierárquica, utilizando-se das variáveis do modelo: v4_bidig, v5_cobibli, v6_publiof, v12_tic, v7_rh, v10_inv. Todos os dados foram analisados de forma integrada, por meio de software estatístico, sem a identificação dos respondentes, reduzindo-se, assim, o viés de interpretação muitas vezes existente em pesquisas dessa natureza.

\section{Resultados}

Dos 24 ministérios, pertencentes à administração pública federal direta, foco desta pesquisa, a maioria possui biblioteca (14) e/ou centro de documentação (4). Esse é um fator positivo, pois essa variável representa um fator crítico de sucesso da infraestrutura informacional que facilita a implantação de biblioteca "em nuvem". Entretanto, dois ministérios não responderam a pesquisa, sendo assim, a partir desse momento, somente serão consideradas as respostas das 16 instituições que possuem ou biblioteca ou centro de documentação que responderam a pesquisa.

Verificou-se que, apenas $18,8 \%$ dessas instituições acreditavam estar preparadas para lidar com a explosão informacional digital por meio da computação "em nuvem". Entretanto, $56,3 \%$ demostram a importância dessa questão ao responderem que estão avaliando o problema.

Outra forma de checar o objetivo principal da pesquisa foi verificar a existência de infraestrutura informacional de TI e de pessoal para lidar com a explosão informacional digital por meio da computação em nuvem, bem como se haverá investimentos para tal.

Sendo assim, o primeiro quesito para o funcionamento da biblioteca "em nuvem" é a existência de uma infraestrutura informacional propicia. Para tanto foram analisadas as seguintes variáveis: v4_bidig, v5_cobibli, v6_publiof. 
A existência de biblioteca digital (v4_bidig) na organização é um dos elementos que contribui com a implantação da biblioteca "em nuvem", visto que os documentos já se encontram em formato digital e organizados. Entretanto, é também necessário que as publicações oficiais estejam armazenadas, em sua totalidade, em biblioteca digital (v6_publiof). Apenas 18,8\% dessas organizações movimentam-se nesse sentido, sendo que somente uma possui a totalidade das publicações oficinas em biblioteca digital. A moda foi não possuir, mas o serviço encontrava-se em estudo em $37,5 \%$ da amostra, com previsão de investimento nos próximos 12 meses.

Ainda no campo da infraestrutura informacional, destacam-se os resultados quanto à existência de controle bibliográfico na instituição (v5_cobibli). Apenas 31,3\% dos respondentes possuíam controle bibliográfico e representavam a moda das respostas. Sem um controle bibliográfico fica mais difícil a implantação da biblioteca "em nuvem", pois a organização não possui o controle da produção bibliográfica institucional ao longo do tempo. De qualquer forma, pelo menos $43,8 \%$ pensavam em investir nesta área.

Outro quesito importante do modelo é a existência de infraestrutura de $\mathrm{TI}$, incluindo plataforma e software como serviço, para o funcionamento da biblioteca "em nuvem. Nesse contexto, verificou-se que somente um elemento amostral possuía serviços informacionais em nuvem" (v8_senuv). Os serviços disponibilizados eram (v9_tipos): ebooks em domínio público; acervo da memória institucional, da legislação institucional e dos documentos do órgão. $\mathrm{O}$ acesso a esses dados poderia ser feito por meio de qualquer dispositivo digital, como smartphones e tablets. Destaca-se, entretanto que, $62,5 \%$, apesar de não disponibilizarem tal serviço, estavam estudando a possibilidade de implantação.

Não havia biblioteca que compartilhasse em nuvem a infraestrutura de tecnologia da informação e comunicação (v12_tic). Entretanto, 43,8\% dos entrevistados estavam avaliando essa possibilidade. Destaca-se que cinco bibliotecas responderam que não dispunham de conhecimento a respeito do assunto.

Caso o sistema de serviço "em nuvem" já estivesse instalado e disponível, as questões consideradas prioritárias para sua utilização, pois apresentaram a maior frequência de respostas (50\%), foram: (1) segurança; e (2) processo de aquisição de equipamentos e software ser demorado e a compra por menor preço nem sempre garante a melhor qualidade da tecnologia adquirida.

Outro quesito importante para a implantação e gestão da biblioteca "em nuvem" é a existência de recursos humanos capacitados basicamente nas três áreas do conhecimento listadas a seguir: biblioteconomia, arquivologia e computação (v7_rh). Nesse contexto, menos 
da metade dessas organizações $(43,8 \%)$ possuía esses profissionais capacitados para atuar na área. Destaca-se que apenas em 12,5\% possuíam essas três modalidades de competência (Tabela 1).

Tabela 1 - Recursos Humanos competentes para trabalhar com biblioteca em nuvem

\begin{tabular}{|c|c|c|c|c|c|c|}
\hline \multirow{3}{*}{ Área } & \multicolumn{5}{|c|}{$\begin{array}{l}\text { Existência de recursos humanos preparados para } \\
\text { trabalhar com biblioteca "em nuvem" }\end{array}$} & \multirow{3}{*}{ Total } \\
\hline & \multicolumn{3}{|c|}{ Sim } & \multicolumn{2}{|c|}{ Não } & \\
\hline & A & B & C & D & $E$ & \\
\hline Social & 0 & 2 & 0 & 0 & 2 & 4 \\
\hline Econômica & 1 & 0 & 1 & 0 & 0 & 2 \\
\hline Defesa e segurança & 0 & 0 & 0 & 2 & 0 & 2 \\
\hline Infraestrutura & 0 & 1 & 0 & 0 & 4 & 5 \\
\hline Estratégia e gestão & 0 & 1 & 1 & 0 & 0 & 2 \\
\hline Ciência e tecnologia & 0 & 0 & 0 & 0 & 1 & 1 \\
\hline Total & 1 & 4 & 2 & 2 & 7 & 16 \\
\hline$\%$ & $6,3 \%$ & $25,0 \%$ & $12,5 \%$ & $12,5 \%$ & $43,8 \%$ & $100,0 \%$ \\
\hline
\end{tabular}

Obs.: (A) Existe na área de tecnologia.

(B) Existe na biblioteca e/ou arquivo.

(C) Existe nas duas áreas acima citadas.

(D) Não existe, mas encontra-se em estudos preliminares.

(E) Não existe e não há estimativa de investimento.

Entretanto, o dado que chamou mais atenção foi o fato de que $43,8 \%$ das instituições não possuíam estimativa de investimento, quesito esse que se caracteriza como um dos fatores críticos de sucesso para implantação da biblioteca "em nuvem".

A existência de recursos financeiros é outro fator crítico de sucesso no processo de implantação da biblioteca "em nuvem" (v10_inv). Entretanto, somente 25\% dos entrevistados possuíam previsão de investimentos permanente para ampliação de espaço de 
armazenamento em função da crescente quantidade de documentos digitais para a preservação da memória institucional.

Avaliando-se as variáveis que sinalizam se a administração pública federal direta está preparada para lidar com a explosão informacional por meio da computação "em nuvem", verificou-se que não. Somente $18,8 \%$ possuíam biblioteca digital (Tabela 1), bem como o mesmo percentual representou o número de instituições que se consideraram preparadas para a explosão do volume informacional digital por meio do Cloud Computing.

Outra forma de identificar-se o quanto a administração pública federal direta está preparada para lidar com a explosão informacional por meio da computação "em nuvem" foi analisando-se os resultados da análise de cluster apresentados na Figura 1 e na Tabela 2, onde estão destacadas as variáveis do modelo utilizadas. A análise dos resultados sugeriu a existência de quatro grupos distintos em relação ao nível de preparação da administração pública federal direta para lidar com a explosão informacional digital por meio da computação "em nuvem":

- Cluster 1 - grupo de bibliotecas prontas para investir na oferta de produtos por meio de biblioteca "em nuvem", pois tanto infraestrutura informacional, quanto de pessoal e tecnológica, além de orçamento para a realização de tal investimento, atendo os quesitos do modelo proposto, formado por três elementos.

- Cluster 2 - grupo de bibliotecas que possuem infraestrutura a quase todos os quesitos do modelo exceto a infraestrutura informacional, que necessita investimentos.

- Cluster 3 - Necessitam investimentos tanto em infraestrutura informacional quanto de pessoal, mas há previsão de investimentos.

- Cluster 4 - Apesar de possuírem biblioteca, não atendem a nenhum dos quesitos do modelo, e não há previsão de investimentos. 
Figura 1 - Dendograma: resultado do processo de clusterização

\section{Dendrogram using Average Linkage (Between Groups)}

Rescaled Distance Cluster Combine

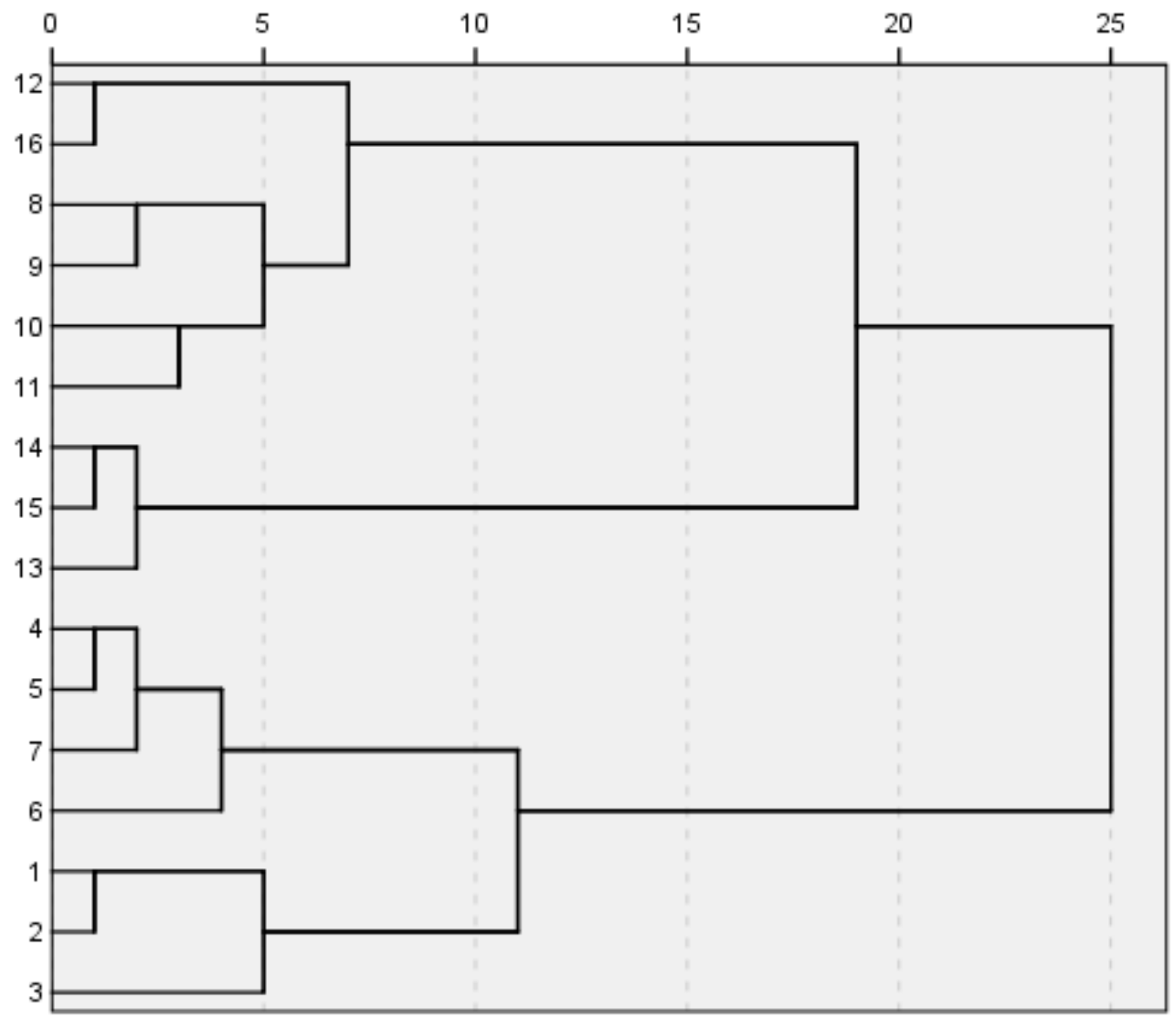

Fonte: Produção dos autores (2014). 
Tabela 2 - Características dos clusters em relação às variáveis chave

\begin{tabular}{|c|c|c|c|c|c|c|}
\hline Amostra & v4_bidig & v5_cobibli & v6_publiof & v7_rh & v10_inv & Cluster \\
\hline 1 & 1 & 1 & 3 & 2 & 1 & 1 \\
\hline 2 & 1 & 1 & 2 & 1 & 2 & 1 \\
\hline 3 & 1 & 1 & 1 & 2 & 2 & 1 \\
\hline 4 & 2 & 2 & 4 & 2 & 1 & 2 \\
\hline 5 & 2 & 2 & 4 & 3 & 2 & 2 \\
\hline 6 & 3 & 3 & 5 & 2 & 2 & 2 \\
\hline 7 & 3 & 3 & 5 & 3 & 2 & 2 \\
\hline 8 & 3 & 1 & 5 & 5 & 2 & 3 \\
\hline 9 & 4 & 2 & 4 & 6 & 2 & 3 \\
\hline 10 & 3 & 3 & 5 & 6 & 1 & 3 \\
\hline 11 & 3 & 1 & 4 & 6 & 1 & 3 \\
\hline 12 & 4 & 4 & 6 & 6 & 2 & 3 \\
\hline 16 & 3 & 4 & 5 & 6 & 2 & 3 \\
\hline 13 & 4 & 4 & 4 & 6 & 6 & 4 \\
\hline 14 & 4 & 3 & 5 & 5 & 6 & 4 \\
\hline 15 & 4 & 4 & 6 & 6 & 6 & 4 \\
\hline
\end{tabular}

Fonte: Produção dos autores (2014).

\section{Conclusões e Recomendações}

A utilização da tecnologia "Cloud Computing" tem crescido, configurando-se como um novo paradigma para o compartilhamento de dados e serviços. Baseia-se na visão SaaS, PaaP e laaS, e se mostra como alternativa para lidar com a explosão de informação digital a custos reduzidos.

São requisitos para a sua implantação e gestão: a existência de infraestrutura informacional digital e tecnológica, bem como pessoal capacitado com perfil multidisciplinar e recursos financeiros para implantação e custeio. 
Essa nova tecnologia apresenta-se como solução para melhoria dos serviços informacionais na Administração Pública Federal Direta que enfrenta a explosão informacional digital. Entretanto, a maioria de suas bibliotecas e centros de documentação não está preparada para atuar no modelo "Cloud Computing". Somente um dos respondentes da pesquisa afirmou possuir serviços em "Cloud Computing" e duas em condições de implantá-la - elementos esses pertencentes ao cluster 1 . Há um grupo da amostra, formado por quatro elementos pertencentes ao cluster 2 , que estão próximos de estarem preparados para a implantação dessa nova tecnologia, possuindo como ponto fraco a estrutura informacional.

Em geral, tanto a infraestrutura informacional quanto de TIC são precárias. Somente $18,8 \%$ da amostra possuem as publicações oficiais armazenadas em biblioteca digital e em $32,3 \%$ há controle bibliográfico e não há bibliotecas ou centros de documentação que compartilhem em nuvem a infraestrutura de TIC. Há também necessidade de investimentos em capacitação de pessoal e recursos para investimento e custeio de todo o processo.

Destaca-se que, o fato das bibliotecas e centros de documentações terem sido o foco dessa pesquisa pode ser considerado como restrição, reduzindo a identificação da capacidade real da instituição de implantar serviços de "biblioteca em nuvem". Entretanto, a fragilidade da infraestrutura informacional levantada não invalida a conclusão da pesquisa.

Como contribuição científica dessa pesquisa, destaca-se a definição dos quesitos para implantação de "biblioteca em nuvem" para as publicações oficiais editadas ou produzidas pela Administração Pública Federal Direta. Esses quesitos são: Infraestrutura informacional; infraestrutura tecnológica; infraestrutura de pessoal; recursos financeiros para investimento e custeio das "bibliotecas em nuvem".

A pesquisa também mostra para as organizações as vantagens do investimento em "Cloud Computing", os quesitos a serem observados para o sucesso de sua implantação. Também chama a atenção que apesar das diversas vantagens, há riscos quanto à segurança da informação.

Recomendam-se futuras pesquisas que definam as competências profissionais necessárias para implantação e gestão de "biblioteca em nuvem"; aperfeiçoamento do modelo de segurança da informação que garanta a confiabilidade dos dados armazenados nesse tipo de biblioteca, o desenvolvimento de método de implantação de biblioteca em nuvem para a Administração Pública Federal Direta, bem como desenvolvimento de modelo de comunicação e marketing para divulgação desses novos serviços para a sociedade. 


\section{Referências}

AVANCINI, Marta. A importância da preservação dos acervos digitais. Disponível em: < http://www.comciencia.br/comciencia/?section=8\&edicao=79\&id=977>. Acesso em: 07 out. 2014.

BELLUZZO, Regina Célia Baptista. As competências do profissional da informação nas organizações contemporâneas. Revista Brasileira de Biblioteconomia e Documentação, Nova Série, São Paulo, v. 7, n. 1, p. 58-73, jan./jun. 2011.

BOERES, Sonia Araújo de Assis. Política de preservação da informação digital em bibliotecas universitárias brasileiras. 2004. 167f. Dissertação (Mestrado em Ciência da Informação e Documentação) - Faculdade de Economia, Administração, Contabilidade e Ciência da Informação e Documentação, Universidade de Brasília, Brasília.

BOSS, Greg; MALLADI, Padma; et al. Cloud Computing. Armonk: IBM, 2007.

BRASIL. Presidência da República. Publicações oficiais brasileiras: guia para editoração. Brasília: Presidência da República, 2010. 220p.

BRASIL. Lei no 12.527, de 18 de novembro de 2011. Regula o acesso a informação. Diário Oficial [da República Federativa do Brasil], Brasília, DF, n. 221-A, 18 nov. 2011. Seção I, p. 1-5.

FOX, E. A.; MARCHIONINI, G. Toward a Worldwide Digital Library. Communications of the Association for Computing Machinery, v. 41, n. 4, p. 29-32, Apr. 1998.

HEYES, Biran. Cloud Computing. Communications of the ACM, v. 51, n. 7, Jul. 2008.

MADHUBALA, R. An illustrative study of cloud computing. International Journal of Software and Engeneering, v. 1, n. 6, Jan. 2012.

MELL, Peter; GRANCE, Tim. The NIST definition of cloud computing. 2009. Disponível em: <http://www.nist.gov/itl/cloud/upload/cloud-def-v15.pdf>. Acesso em: 10 jun. 2014.

MOUTINHO, Karina; CUNHA FILHO, Paulo C.; LIMA, Alessandra Marques de. Liber: alternativa para publicação eletrônica. Ciência da Informação, Brasília, v. 31, n. 2, p. 80-85, maio/ago. 2002.

MULER, Diane. Law libraries in the cloud. Law Library Journal, v. 101, n. 2, 2012.

PASQUI, V. II cloud computing e le biblioteche: illusione o opportunità? JLIS.it, v. 1, n. 2, p. 277-304, Dec. 2010.

MUELEY, Diane. Law libraries in the cloud. Law Library Journal, v. 101, n. 2, p. 249-254, 2009. PLUMMER, et al. Cloud Computing: Defining and Describing an Emerging Phenomenon. Gartner, $2008 . \quad$ Disponível em: <http://www.emory.edu/BUSINESS/readings/CloudComputing/Gartner_cloud_computing_def ining.pdf>. Acesso em 21 set. 2014.

SANCHEZ; Otavio Prospero; CAPPELLOZZA, Alexandre. Antecedentes da adoção da computação em nuvem: efeitos da infraestrutura, investimento e porte. Revista de Administração Contemporânea, v. 16, n. 5, art. 1, p. 646-663, set./out. 2012.

SARACEVIC, Tefko. Interdisciplinary nature of information science. Ciência da informação, Brasília, v. 24, n. 1, p. 36-41, 1995. 
SANTOS, C.J.O.; CAVALCANTE, R.S.; GALVÃO, A.I.C. Controle bibliográfico de publicações oficiais: uma proposta para o cenário brasileiro. In: CONGRESSO BRASILEIRO DE BIBLIOTECONOMIA, DOCUMENTAÇÃO E CIÊNCIA DA INFORMAÇÃO, 25., 2013, Florianópolis. Anais... São Paulo: FEBAB, 2013.

SHOWER, Ben. Saving libraries: the battle for time and resource. JISC Inform, v. 32, p.8-19, 2011. Disponível em: <http://www.jisc.ac.uk/inform/inform32/SavingLibraries.html>. Acesso em: 21 set. 2014.

SILVA, J. F. Modesto da; MUCHERONI, M. L. As Bibliotecas em nuvens. In: CONGRESSO BRASILEIRO DE BIBLIOTECONOMIA, DOCUMENTO E CIÊNCIA DA INFORMAÇÃO, 25, 2013, Florianópolis. Anais... $\quad$ Florianópolis, $2013 . \quad$ Disponível em: <http://portal.febab.org.br/anais/article/view/1383>. Acesso em: 21 de out. 2014.

SOUTO, S. M.de O. O profissional da informação frente às tecnologias do novo milênio e as exigências do mundo do trabalho. 4, CINFORM. Anais... 2006. Disponível em: <http://www.cinform.ufba.br/iv_anais/artigos/texto16.html>. Acesso em: 14 jul. 2014

ROSETTO, M.; NOGUEIRA, A. H. Aplicação de elementos metadados Dublin Core para descrição de dados bibliográficos on-line da biblioteca digital de teses da USP. In: SEMINÁRIO NACIONAL DE BIBLIOTECAS UNIVERSITÁRIAS, 12., 2002, Recife. Anais... Recife: UFPe, 2002. 1 CD-ROM.

THIBODEAU, Kenneth. Overview of Technological Approaches to Digital Preservation and Challenges in Coming Years. Disponível em: <http://www.clir.org/pubs/reports/pub107/thibodeau.html>. Acesso em: 1 jul. 2014.

TOFFOLI, Ticiana Oniki. Especificação de bibliotecas digitais de objetos complexos. 2012. 103 f. Dissertação (Mestrado em Ciência da Computação) - Universidade Estadual de Campinas, Campinas, 2012.

VOAS, Jeffrey; ZHANG, Jia. Cloud Computing: New wine or just a new bottle? IEEE Computer Society, 2009.2 Disponível em: <http://www.computer.org/csdl/mags/it/2009/02/mit2009020015.html>. Acesso em: 21 set. 2014.

YUVARAJ, Mayank. Cloud computing applications in Indian Central University libraries: a study of librarians' use. Library Philosophy and Practice, 2013. Disponível em: $<$ http://digitalcommons.unl.edu/cgi/viewcontent.cgi?article=2397\&context=libphilprac $>$. Acesso em: 10 jun. 2014.

YUVARAJ, Mayank; SINGH, Ajay Pratap. Cloud computing in conjunction with libraries: Descriptive literature review. Asia Pacific of Library and Information Science, v. 2, n. 2, p. 86107, Jul./Dec. 2012.

Recebido/Recibido/Received: 2015-07-31

Aceitado/Aceptado/Accepted: 2015-10-08 